\title{
¿LIBERTAD DE EXPRESIÓN O RESPONSABILIDAD? ¿CUÁNDO, EN EL CASO DE PORTALES SOBRE NOTICIAS, DE ACUERDO CON LA CORTE EUROPEA DE DERECHOS HUMANOS? ANÁLISIS Y LECTURAS ACTUALES*
}

Víctor Manuel CoLLí EK**

\section{INTRODUCCIÓN. ¿LIBERTAD O RESPONSABILIDAD?}

Reconocer la responsabilidad de un portal de noticias sobre la posible afectación de derechos es entendible, pero ¿hasta dónde un portal web de noticias puede ser considerado responsable, no por la noticia colocada, sino por los comentarios que se hicieron sobre la misma? Es una pregunta interesante porque ¿el portal se encontrará protegido por su libertad de expresión, si esos comentarios afectan el derecho a la vida privada de la persona, a la que hace referencia la noticia?

* Este trabajo, realizado durante la estancia de investigación en Washington D. C. de noviembre a diciembre de 2013, es producto del proyecto de investigación: "La vigencia de la Constitución en la interpretación de la Suprema Corte de Justicia de la Nación como Tribunal Constitucional”, 154998, Conacyt-SEP, Ciencia Básica, del cual soy responsable. Agradezco la colaboración de la becaria Mariana Guadalupe Casas Collí, sin la cual no hubiera sido posible la conclusión de este documento.

** Profesor-investigador en el Centro de Investigaciones Jurídicas de la Universidad Autónoma de Campeche; profesor del Promep-SEP; miembro del Sistema Nacional de Investigadores; publicaciones recientes: "Improving Human Rights in Mexico. Constitutional Reforms, International Standards, and New Requirements for Judges", Human Rights Brief, American University Washington College of Law, y "Federalismo judicial en México. Concepciones, evolución y perspectivas”, Revista de Estudios Autonómicos y Federales, Barcelona. 
Esas preguntas estuvieron en el centro de la decisión Delfi contra Estonia, resuelta el 10 de octubre de 2013, por la primera sección de la Corte Europea de Derechos Humanos (CoEDH). ${ }^{1}$

El caso analizaba la responsabilidad de un sitio de noticias de Internet, por los comentarios ofensivos y anónimos que fueron posteados por los lectores de uno de los artículos que habían sido colgados. Es importante indicar que éste es el primer caso de la CoEDH sobre la responsabilidad de los proveedores de Internet. ${ }^{2}$ En ese sentido, el sitio Delfi consideraba violada su libertad de expresión - contenido en el artículo 10 de la Convención Europea de Derechos Humanos (CEDH) - al definirse su responsabilidad, por comentarios de sus lectores. ${ }^{3}$

\section{DeSENVOLVIMIENTO PREVIO A LA CORTE Europea DE DERECHOS Humanos}

Delfi $\mathrm{AS}^{4}$ es una compañía que posee uno de los más grandes sitios de Internet de noticias de Estonia. El 24 de enero de 2006 fue publicada en el portal una nota denominada SLK Destroyed Planned Roads acerca de una

1 Sentencia Delfi vs. Estonia (solicitud núm. 64569/09), http://hudoc.echr.coe.int/ sites/fra/pages/search.aspx?i=001-126635.

2 CFR, http://www.husovec.eu/2013/10/echr-on-liability-of-isps-as.html. Igualmente, Frosio, Giancarlo, "The European Court of Human Rights Holds Delfi.EE Liable for Anonymous Defamation", 25 de octubre de 2013, http://cyberlaw.stanford.edu/blog/2013/10/ european-court-human-rights-holds-delfiee-liable-anonymous-defamation.

3 Este artículo señala: "1. Toda persona tiene derecho a la libertad de expresión. Este derecho comprende la libertad de opinión y la libertad de recibir o de comunicar informaciones o ideas sin que pueda haber injerencia de autoridades públicas y sin consideración de fronteras. El presente artículo no impide que los Estados sometan a las empresas de radiodifusión, de cinematografía o de televisión a un régimen de autorización previa. 2. El ejercicio de estas libertades, que entrañan deberes y responsabilidades, podrá ser sometido a ciertas formalidades, condiciones, restricciones o sanciones, previstas por la ley, que constituyan medidas necesarias, en una sociedad democrática, para la seguridad nacional, la integridad territorial o la seguridad pública, la defensa del orden y la prevención del delito, la protección de la salud o de la moral, la protección de la reputación o de los derechos ajenos, para impedir la divulgación de informaciones confidenciales o para garantizar la autoridad y la imparcialidad del poder judicial", http://www.echr.coe.int/ Documents/Convention_SPA.pdf.

4 Véase igualmente Article 19, Statement, "European Court Strikes Serious Blow to Free Speech Online", 14 de octubre de 2013, http://www.article19.org/resources.php/ resource/37287/en/european-court-strikes-serious-blow-to-free-speech-online. 
compañía de Ferry de "L", el accionista mayoritario de esa compañía, donde se discutía el cambio de ruta a determinadas islas, lo que había resultado en el resquebrajamiento del hielo, atrasando por semanas la apertura de lo que podían haber sido carreteras o vías públicas sobre el hielo, que se consideraban más rápidas y baratas que el Ferry. En dos días, 24 y 25 de enero, se alcanzaron 185 comentarios, de los cuales alrededor de 20 contenían amenazas personales y lenguaje ofensivo contra " $L$ ".

Algunos de los comentarios que se postearon a la noticia fueron: ${ }^{5}$

1. There are currents in [V]äinameri. 2. Open water is closer to the places you referred to, and the ice is thinner.

Proposal - let's do as in 1905, let's go to [K] uressaare with sticks and put [L.] and [Le.] in bag

2. Fucking shitheads...

They bath in money anyways thanks to that monopoly and State subsidies and now started to fear that cars may drive to the islands for a couple of days without anything filling their purses. Burn in your own ship, sick Jew!

3. Good that [La.'s] initiative has not broken down the lines of the web flamers. Go ahead, guys, [L.] into oven!

4. [Little L.] go and drown yourself

5. Aha... [I] hardly believe that that [happened] by accident... assholes of fuck.

Abajo de la nota, los usuarios - teniendo acceso a comentarios de otros usuarios - en algunas de sus respuestas, habían utilizado palabras altamente ofensivas sobre el operador y el dueño del ferri.

El 8 de marzo de 2006, los abogados de "L" solicitaron al sitio el retiro de los comentarios y la suma de 32,000 euros en compensación por daños no pecuniarios. El mismo día los comentarios fueron retirados y el 23 de marzo de 2006, la compañía del portal argumentó que esto último lo había hecho bajo la obligación del sistema de notice-and-take-down y que se negaba a los daños. El dueño demandó a Delfy en 2006, logrando sentencia favorable en 2008. Lo anterior fue apelado por la compañía Delfy ante la Suprema Corte de Estonia, indicando que bajo la directiva europea 2000/31/EC, sobre comercio electrónico, su rol como proveedor de servicios de Internet había sido técnica, pasiva y neutral, y esto lo eximía de responsabilidad.

5 Cfr. Delfi vs. Estonia, párr. 10. 
La Corte determinó que Delfi había ejercido control sobre la publicación de los comentarios, y esto lo excluía de tal eximente. ${ }^{6}$

\section{LAS DISPOSICIONES SOBRE LA SOCIEDAD \\ DE LA INFORMACIÓN}

Delfi argumentaba que su responsabilidad estaba exenta, porque debía ser considerado un proveedor de servicios informáticos con esta eximente, tal como se encontraba reconocido en las leyes sobre sociedad de información, tanto a nivel europeo como del país. ${ }^{7}$

Bajo estas leyes de la sociedad de la información, el proveedor de servicios es un técnico que no tiene conocimiento o control sobre la información. En todas las directivas relativas a la sociedad de la información se subraya el hecho de la labor neutral del proveedor del servicio — entendida ésta como técnica, automática, pasiva, y sin ningún conocimiento o control sobre los datos-.

Lo anterior ha sido igualmente determinado por la Corte de Justicia de la Unión Europea en diversos casos: en el caso Google France and Google del 23 de marzo de $2010^{8}$ se determinó que bajo el artículo 14 de la Directiva 2000/31, debía subrayarse el carácter neutral del proveedor de servicios de Internet; en el caso L'Oreal and Other ${ }^{9}$ el 12 de julio de 2011 se estableció la excepción de "neutralidad" en un operador que desempeña un rol en el mejoramiento de los datos, para hacerlos vendibles o atrayentes; en el caso Scarlett Extended del 24 de noviembre de 2011, ${ }^{10}$ finalmente se decidió la neutralidad, cuando un proveedor de servicios es compelido a colocar un software de control peer-to-peer, para controlar el tráfico de información dentro de una red que aplique indiscriminadamente a todos los clientes, por un tiempo ilimitado, exclusivamente preventivo y a sus expensas, a fin de

6 Cfr. Johan, Tere, "Estonian Supreme Court ruled in Favor of Leedo in the Dispute against Delfi", 10 de junio de 2009, http://www.baltic-course.com/eng/Technology/? $d o c=14708$.

7 Entre otros: 1. Information Society Services Act, ley nacional que adoptaba las directivas europeas; 2. Directive 2000/31/EC, del Parlamento y Consejo europeos; 3. Directive 98/34/EC, igualmente del Parlamento y Consejo europeos.

8 http://curia.europa.eu/juris/liste.jsf?num $=C-236 / 08$.

9 http://curia.europa.eu/juris/liste.jsf?language $=$ en\&num $=C-324 / 09$.

10 http://curia.europa.eu/juris/liste.jsf?language $=$ en\&num $=C-70 / 10$. 
vetar la transmisión de datos con derechos reservados, relativos a música, películas, etcétera. En ese sentido, para determinar la responsabilidad bajo este aspecto, debía determinarse la neutralidad del portal en la colocación de los comentarios.

\section{EL SISTEMA DE CONTROL DE COMENTARIOS \\ DEL PORTAL}

Como hemos dicho líneas arriba, el problema a dilucidar era la responsabilidad del portal sobre los comentarios realizados a una noticia. En ese sentido, debía analizarse cómo se controlaban estos comentarios para determinar el grado de participación del portal en los mismos.

En la lectura de la sentencia encontramos, a grandes rasgos, la forma en que operaba este sistema. Abajo de las noticias se encontraba un campo de add your comment, con la opción de colocar el nombre y el email del comentador. Se podía acceder a los otros comentarios en un campo aparte read comments, los cuales eran colgados de manera automática, sin algún tipo de edición o moderación por parte del dueño del sitio. Los comentarios podían ser posteados anónimamente, porque no se exigía la identificación.

La página tenía un sistema de triple control de los comentarios. El primero notify-and-take-down, donde un comentador podía marcar de inapropiado un comentario, lo que generaría su borrado. Segundo, un sistema automático de filtrado y de borrado al emplear palabras marcadas como obscenas. Tercero, una víctima de comentarios difamatorios podía notificar al administrador, en cuyo caso el comentario sería borrado automáticamente.

Adicionado a lo anterior, Delfi tiene una política pública sobre la autoría de los comentarios, subrayando que son responsabilidad de quienes los emiten y no de Delfi, al igual que una serie de reglas sobre los comentarios.

\section{Resolución de la Corte Europea de Derechos Humanos}

En su resolución, la CoEDH estudió cuatro elementos:

a) El contexto de los comentarios.

b) Las medidas tomadas por Delfi para prevenir los comentarios difamatorios. 
c) Si se podrían haber demandado a los autores originales de los comentarios.

d) Las consecuencias de haber hecho responsable a Delfi.

La compañía argumentaba que reconocía el daño hecho a "L" por los comentarios, pero que éstos eran responsabilidad de sus autores. Los comentarios no habían sido sugeridos por la compañía y el sistema de seguridad de los comentarios two-limb system era suficiente para considerar su responsabilidad excluida.

Argumentaba que no había jugado un rol activo en los comentarios, sino solamente había sido depósito de los mismos. Haciendo para esto referencia a la Declaración y al artículo 15 de la Directiva de Comercio Electrónico de la Unión Europea.

La Corte argumentaría que, en el caso concreto, se trataba de un administrador que tenía inclusive control sobre el "ambiente de los comentarios", ya que invitaba a hacerlos, existía una política de edición de los mismos que él controlaba, porque por ejemplo, los comentaristas mismos no podían borrar directamente las notas que ellos mismos posteaban, sólo podían denunciar los considerados obscenos, esto reforzaba la idea que el administrador del sitio sí tenía control sobre los comentarios. El gobierno argumentó que Delfi había ejercido control parcial. Al igual que subrayó que Estonia había reducido la respuesta estatal a los casos de difamación, de la dimensión penal a la civil, como había sido aplicado al caso. No se podía considerar el caso de un proveedor de servicios de almacenado, bajo la ley del servicio de la sociedad de la información, porque en tal caso, inclusión y borrado de la información es facultad del cliente, pero por las circunstancias, Delfi se consideraba más bien un proveedor de servicios de contenido.

Dejando esto claro, proseguía el análisis sobre la afectación de la libertad de expresión. Al continuar su examinación sobre este tema, observamos lo siguiente.

\section{La restricción a la libertad de expresión debe encontrarse establecida en la ley}

La Corte empieza subrayando su teoría de que una norma puede ser considerada ley, si es formulada con suficiente precisión para permitir a los ciudadanos regular su conducta, permitiéndole "prevenir" las consecuencias 
que una determinada actividad puede llevar. Reconoce que determinadas leyes pueden ser vagas, mientras que otras más puntuales. En este sentido, el criterio de "prevención" dependerá de las circunstancias. Inclusive, puede significar la necesidad de solicitar consejo. Lo cual es particularmente importante en personas que realizan una actividad profesional.

Esto es importante, porque en el presente caso se consideró que las leyes aplicables - por ejemplo, Information Society Service Act - carecían de detalle. Inclusive, la aplicación de las leyes relativas a actividades periodísticas y editores, a portales de Internet y sus administradores, era novedosa.

La Corte argumentó que Delfi, como un editor profesional, y como uno de los portales de noticias más grande de Estonia, debía al menos estar familiarizado con la legislación y con la jurisprudencia aplicable, al igual que haber pedido apoyo legal, esto significaba que debió conocer los riesgos propios de su actividad y las consecuencias que ésta podría tener. En ese sentido, se cumplía con el requisito de que la restricción a la libertad de expresión debía encontrarse establecida en la ley.

\section{Objetivo legítimo}

¿Cuál era el objetivo de la restricción? La restricción de la libertad de expresión tiene como objetivo proteger la reputación y el derecho de los otros. Ahora, ¿la restricción era necesaria?

\section{Severidad de la interferencia}

Los comentarios fueron difamatorios: sobre esto, dice la Corte, no hay controversia. Otra cuestión no controvertida era que sobre la noticia no había problema. El artículo publicado por el portal de noticias llevaba un cierto grado de interés público.

It discussed a shipping company's moving its ferries from one route to another and in doing so breaking the ice at potential locations of ice roads, as a result of which the opening of such roads - a cheaper and faster connection to the islands compared to the company's ferry services - was postponed for several weeks. ${ }^{11}$ 
Se subrayó que el artículo mismo estaba balanceado y no era motivo de disputa. El desplazamiento del artículo fuera del tema de controversia generaba la problemática planteada por el portal de noticias, porque llevaba a un sencillo razonamiento. La responsabilidad que este portal reconocía era sobre el posteo del artículo, eso había sido decisión del portal, tanto en la decisión del tema como en el de su publicidad. Pero los comentarios hechos a ella, no eran de su autoría, ¿por qué debía ser responsable por ellos? Era aparentemente claro, el portal no había sido autor de los comentarios, y al no serlo, no podría sufrir riesgos de responsabilidad por su contenido. Además, tratándose de un portal de Internet, aplicaban las directivas de la sociedad de la información, que lo eximían de responder por información en la cual no tenía control.

A ese razonamiento, la CoEDH empieza señalando que, aunque el artículo no fue motivo de controversia, su exposición en la página web de noticias sí generaba responsabilidad, por diversos motivos. Primero, el posteo de la nota debió estar acompañado con una conducta necesariamente precautoria, para evitar la adjudicación de una posterior responsabilidad. Basada en la naturaleza del artículo y el historial del portal, se podía determinar que la publicación del mismo generaba un riesgo alto de respuestas negativas que pudieran ir más allá de lo aceptable, ${ }^{12}$ lo que se agravaba con el número de comentarios que generó, igualmente más allá del promedio. El siguiente elemento a estudiar era el sistema de protección de comentarios con que contaba el portal. Se consideraba que no existía una total negligencia, aunque existían insuficiencias, como el filtrado de palabras. El portal contaba con take-and-down-system, y si con éste se satisfacía la debida diligencia del portal, era una parte importante de la controversia. Primero, su funcionamiento era sencillo, se trataba sólo de oprimir un botón, diría la Corte. En el caso concreto, esta herramienta no fue utilizada por el quejoso, se prefirió el envío de un email, para solicitar el borrado, lo que efectivamente sucedió días después, y aquí otro tema de controversia, pues para cuando se borraron: "the comments had already been accessible to the public for six weeks". ${ }^{13}$

12 Idem: "considering the general reputation of comments on the Delfi news portal, there was a higher-than-average risk that the negative comments could go beyond the boundaries of acceptable criticism and reach the level of gratuitous insult or hate speech".

13 Ibidem, párr. 88. 
Avanzado aquí el razonamiento de la Corte, se entremezcla el tema económico. El diseño y funcionamiento de los sistemas de control de comentarios de portal tenía una fuente importante, el patrocinio. Al postear las noticias y dejar ver los comentarios, la publicidad de los mismos establecía una liga causal con la capacidad económica del portal, porque en el "éxito" de la nota a través de sus comentarios, se captaban divisas: "It was interested in the number of readers as well as comments, on which its advertising revenue depended". ${ }^{14}$

Este último argumento fue considerado significativo para medir el grado de interferencia con la libertad de expresión del portal. Lo anterior se traducía en el funcionamiento del sistema. El portal, por supuesto, tenía el conocimiento de qué noticia se iba a publicar, prevenir la naturaleza de los comentarios que se harían sobre ella, y por tanto, tomar las medidas correspondientes para controlar que esos comentarios difamatorios no se hicieran públicos. De hecho, los comentarios una vez hechos, no podían ser modificados por los comentaristas. Esto daba un grado de control significativo al portal, por sobre lo que se comentaba.

Otro elemento que se calificaba como reductor de la severidad de la interferencia de la libertad de expresión, era que sobre el portal no se imponía medida alguna para obligarlo a controlar la personalidad de los comentaristas, cuestiones que pudieren haber sido entre otras: registro de nombre de ellos, monitoreo de los comentarios, revisión rápida de los mismos previos a su publicidad, etcétera. El portal argumentaba que la persona afectada pudo realizar demandas de difamación directamente a los comentaristas, pero esto generaba un problema, porque en muchos casos, los comentaristas no eran identificables, ya que usaban seudónimos, esto también permitido por dicho portal. Lo anterior llevaba a un argumento importante en el balance entre la libertad de expresión y la obligación del Estado de proveer seguridad para los derechos de personalidad. ${ }^{15}$ De entrada, la Corte era consciente del deseo de los comentaristas de presentarse por medio de seudónimos, en el Internet la información una vez hecha pública permanecerá pública y circulará por siempre, esto llama a la precaución. Sin embargo, seguir el

14 Ibidem, párr. 89.

15 Ibidem, párr. 91: "the Court is not convinced that measures allowing an injured party to bring a claim only against the authors of defamatory comments - as the applicant company appears to suggest - would have, in the present case, guaranteed effective protection of the injured person's right to private life". 
argumento del portal podría llevar a la insatisfacción del derecho de personalidad, por falta de justiciabilidad del mismo, ${ }^{16} \mathrm{y}$ era trascendental un ejercicio de ponderación entre las capacidades de la persona difamada para detectar comentarios difamatorios, contra las de la compañía del portal, con mejores recursos para filtrarlos. El portal, al establecer cierto grado de control sobre los contenidos de lo dicho, poseía un grado de responsabilidad.

Por último, la medida de condena fue el pago de 320 euros, por daños no pecuniarios, que al tratarse de uno de los portales de Internet más grandes de Estonia, no debía considerarse desproporcionada.

En pocas palabras, existió una interferencia con la libertad de expresión, pero estaba justificada.

\section{LECTURAS}

El caso Delfi despierta interrogantes importantes para el futuro de la responsabilidad de los portales de Internet frente a comentarios anónimos o inclusive frente a comentarios en general, como dirían algunos expertos. De entrada pone a reflexionar a estos portales sobre la viabilidad de este tipo de comentarios, por temor a resultar afectados en un futuro.

La carga parecería caer sobre el portal, que deberá contar con sistemas más desarrollados para monitorear todos los comentarios hechos sobre sus noticias. ${ }^{17}$ Otro elemento a considerar sería la fuente jurídica de la responsabilidad, de acuerdo con Tim Worstall ${ }^{18}$ los comentarios online no están gobernados por el lugar donde se realizan, ni tampoco por el lugar donde están los servidores que los alojan, sino por el lugar donde se leen, esto lleva a tener que considerar, cada vez que se escribe algo, las leyes aplicables donde esto se pudiera leer, lo cual hace una carga más grande para los portales.

16 En Krone Verlag GmbH \& Co. KG vs. Austria (núm. 4), núm. 72331/01, §32, 9 de noviembre de 2006, la CoEDH definió que: “shifting the defamed person's risk to obtain redress for defamation proceedings to the media company, usually in a better financial position than the defamer, was not as such a disproportionate interference with the media company's right to freedom of expression".

17 Cfr. Reidy, Pradaig, "European rulling Expels Trouble for Online Comment", 10 de octubre de 2013, http://www.indexoncensorship.org/2013/10/european-ruling-spellstrouble-online-comment/.

18 Véase Worstall, Tim, "Every Website that Accepts Comments Now has a European Problem", 10 de noviembre de 2013, http://www.forbes.com/sites/timworstall/2013/10/11/ every-website-that-accepts-comments-now-has-a-european-problem/. 
La decisión llega en un momento donde el valor de las secciones de comentarios está siendo fuertemente debatido en ambos lados del Atlántico. ${ }^{19} \mathrm{Si}$ la CoEDH ha tomado la decisión de hacer responsable a los sitios de Internet, en los EUA no sucede así, debido a la sección 230 de la Communication Decency Act de 1996, la cual prohíbe que los sitios web sean considerados responsables por los contenidos creados por sus usuarios. ${ }^{20}$ El caso más reciente, que confirma esta tendencia en los EUA, es Small Newspaper Group Inc, ${ }^{21}$ donde se consideró que este sitio no podía calificarse de editor por comentarios anónimos en sus sitios, y por tanto no era responsable.

Definitivamente la resolución llega en un momento de gran convulsión para la vida online. No se puede hablar de esto sin hacer referencia al impacto Snowden 22 y sus revelaciones en el mundo digital.

La libertad de prensa es una víctima colateral, que no secundaria, de un escándalo cuyos detalles siguen poniendo al descubierto una imagen casi obsesiva por el control indiscriminado de datos que la NSA habría seguido

19 Jenifer Garnett indica que la decisión: "is a serious blow to freedom of expression... displays a profound failure to understand EU legal framework for intermediary liability [and] conveniently ignores relevant international standards in the area of freedom of expression on the Internet". In contrast, the United States has codified the principle that online portal providers "shall [not] be treated as the publisher or speaker of content provided by a third party", en "European Cour Finds Liability for Defamatory Comments by Anonymous Users", http://jolt.law.harvard.edu/digest/jurisdiction/international-deci sions/european-court-finds-liability-for-defamatory-comments-by-anonymous-users.

20 Cfr. Zara, Christofer, "Websites Liable For Anonymous Comments? European Court Upholds Defamation Case against Estonian News Portal”, 11 de octubre de 2013, http://www.ibtimes.com/websites-liable-anonymous-comments-european-court-upholdsdefamation-case-against-estonian-news.

21 La sentencia es consultable en http://www.state.il.us/court/R23_Orders/Appellate Court/2012/3rdDistrict/3110594_R23.pdf en la explicación sobre lo que se sostuvo en ella encontramos: "The trial court properly dismissed plaintiffs' complaint with prejudice after finding the federal Communications Decency Act provided immunity for defendants who did not qualify as the 'publishers' of anonymous comments posted to a website associated with the news service".

22 Entre otras muchas notas que han aparecido, véase Sontani, Ashkan et al., "NSA uses Google Cookies to Pinpoint Targets for Hacking", The Washington Post, 10 de diciembre de 2013, http://www.washingtonpost.com/blogs/the-switch/wp/2013/12/10/ nsa-uses-google-cookies-to-pinpoint-targets-for-hacking/. 
— por llegar a los ejemplos más pintorescos— incluso a través de juegos en línea como World of Warcraft o Second Life. ${ }^{23}$

Esto necesariamente va aparejado con el problema libertad-privacidadseguridad dentro de la sociedad. El problema Snowden lo que ha hecho es generar un despertar de la discusión, con cada vez más adeptos que respaldan el aseguramiento de la libertad-privacidad y la necesidad de controles a los actores encargados de garantizar la seguridad. Afortunadamente, parece estarse construyendo una verdad social, que da el lugar preeminente a la dignidad. ${ }^{24}$ Primero, el informe del Panel de Revisión de la Casa Blanca que recomienda mayor vigilancia judicial pública y restricciones a la NSA. ${ }^{25}$

Segundo, la sentencia del juez Richard León, quien diría: "No puedo imaginar una mayor 'indiscriminada' y 'arbitraria invasión', que esta sistemática y altamente tecnológica recolección y retención de datos personales en virtualmente cada ciudadano particular, para los propósitos de consultar y analizarla sin aprobación judicial"' ${ }^{26}$ Tercero, la resolución unánime de la ONU — The Right to Privacy in a Digital Age — ${ }^{27}$ donde los 193 países que la integran, favorecen una decisión que exige la garantía y límite del espionaje en las telecomunicaciones e Internet.

Una pregunta obligada es ¿en un mundo orweliano, ${ }^{28}$ cómo seguimos planteándonos el tema de las responsabilidades? Sobre todo porque se trata de definir la responsabilidad corporativa, ahí tenemos dos respuestas diferentes en "ambos lados del Atlántico". Aunque esto no queda totalmente a manos del Estado, la famosa "carta de recomendación"29 enviada por los

23 "Víctimas colaterales", sección Editorial, El País, 11 de diciembre de 2013, http:// elpais.com/elpais/2013/12/10/opinion/1386700261_735049.html.

24 Cfr. Collí Ek, Víctor Manuel, "La injusticia del derecho", sección Opinión, Reforma, México, 10 de enero de 2014, p. 15.

25 Caño, Antonio, "Obama promete una reforma del espionaje para hacerlo más confiable", El País, 20 de diciembre de 2013, http://internacional.elpais.com/internacio nal/2013/12/20/actualidad/1387572220_003183.html.

26 http://www.theguardian.com/world/2013/dec/16/nsa-phone-surveillance-likelyunconstitutional-judge.

27 Véanse las discusiones en https://www.un.org/News/Press/docs/2013/gashc4094. doc.htm.

28 Cfr. Collí Ek, Víctor Manuel, "Humanizar al Gran Hermano", Tribuna de Campeche, 1o. de diciembre de 2013, http://tribunacampeche.com/2013/12/derecho-la-privacidad/.

29 La página web donde concentraron esta propuesta de nueva política, "Global Government Surveillance Reform”, http://reformgovernmentsurveillance.com/. En ella en- 
tech giants ${ }^{30}$ al gobierno de los EUA, implican que el tema de las responsabilidades y sus efectos — para ellos principalmente económicos $-{ }^{31}$ deben cambiar, las reglas del juego deben cambiar.

La desavenencia en esos "lados" deja ver con claridad la naturaleza conflictiva de este tema, que seguro se acrecentará al enfrentar los retos actuales.

\section{BIBLIOGRAFÍA}

Article 19, Statement, "European Court Strikes Serious Blow to Free Speech Online", 14 de octubre de 2013, http://www.article19.org/re sources.php/resource/37287/en/european-court-strikes-serious-blowto-free-speech-online (consultado el 8 de diciembre de 2014).

CAÑO, Antonio, "Obama promete una reforma del espionaje para hacerlo más confiable", El País, 20 de diciembre de 2013, http://internacional.el pais.com/internacional/2013/12/20/actualidad/1387572220_003183. html (consultado el 8 de diciembre de 2014).

Collí EK, Víctor Manuel, "Humanizar al Gran Hermano", Tribuna de Campeche, 1o. de diciembre de 2013, http://tribunacampeche.com/2013/12/ derecho-la-privacidad/ (consultado el 8 de diciembre de 2014). , "La injusticia del derecho", sección Opinión, Reforma, México, 10 de enero de 2014, p. 15.

Corte Europea de Derechos Humanos, Primera Sección, Caso Delfi AS vs. Estonia (solicitud núm. 64569/09), http://hudoc.echr.coe.int/sites/

contramos cinco principios de esta nueva política: "1. Limiting Governments' Authority to Collect Users' Information. 2. Oversight and Accountability. 3. Transparency about Government Demands. 4. Respecting the Free Flow of Information. 5. Avoiding Conflicts among Governments".

30 Cfr. Wyatt, Eduard y Miller, Claire, "Tech Giants Issue Call for Limits on Government Surveillance of Users", The New York Times, 9 de diciembre de 2013, http://www. nytimes.com/2013/12/09/technology/tech-giants-issue-call-for-limits-on-governmentsurveillance-of-users.html? smid $=f b$-share\&_ $r=0$.

31 "La campaña propone restringir la capacidad de los Estados para pedir a las empresas datos sobre sus usuarios, ya que se está dañando 'la confianza' que los clientes tienen en Internet, lo que según un estudio citado por el diario The New York Times podría costar a la industria para 2016 hasta 180,000 millones de dólares en pérdidas, una cuarta parte de sus ganancias", en Monge, Yolanda, "Las mayores empresas de Internet piden a EEUU regular las leyes de espionaje", El País, 9 de diciembre de 2013, http://internacional. elpais.com/internacional/2013/12/09/actualidad/1386588911_791315.html. 
fra/pages/search.aspx? $i=001-126635$ (consultado el 8 de diciembre de 2014).

-, Primera Sección, Krone Verlag GmbH \& Co. KG vs. Austria (núm. 4), núm. 72331/01, §32, 9 de noviembre de 2006, http://hudoc. echr.coe.int/sites/eng/pages/search.aspx? $i=001-61538$ (consultado el 8 de diciembre de 2014).

Directiva 2000/31/EC del Parlamento Europeo y del Consejo, 8 de junio de 2000, relativa a determinados aspectos jurídicos de los servicios de la sociedad de la información, en particular el comercio electrónico en el mercado interior (Directiva sobre el Comercio Electrónico), http:// eur-lex.europa.eu/LexUriServ/LexUriServ.do? uri=CELEX:32000 L0031:Es:HTML (consultado el 8 de diciembre de 2014).

Directiva 98/34/EC, por la que se establece un procedimiento de información en materia de las normas y reglamentaciones técnicas y de las reglas relativas a los servicios de la sociedad de la información, del Parlamento y Consejo Europeos, http://ec.europa.eu/enterprise/tris/conso lidated/index_es.pdf(consultado el 8 de diciembre de 2014).

Frosio, Giancarlo, "The European Court of Human Rights Holds Delfi. EE Liable for Anonymous Defamation", 25 de octubre de 2013, http:// cyberlaw.stanford.edu/blog/2013/10/european-court-human-rightsholds-delfiee-liable-anonymous-defamation (consultado el 8 de diciembre de 2014).

GARNETT, Jenifer, "European Cour Finds Liability for Defamatory Comments by Anonymous Users", 19 de octubre de 2013, http://jolt.law. harvard.edu/digest/jurisdiction/international-decisions/europeancourt-finds-liability-for-defamatory-comments-by-anonymous-users (consultado el 8 de diciembre de 2014).

"Global Government Surveillance Reform", http://reformgovernmentsur veillance.com/ (consultado el 8 de diciembre de 2014).

HuSOVEC, Martin, "ECHR on Liability of ISPs as a Restriction of Freedom of Speech", 14 de octubre de 2013, http://www.husovec.eu/2013/10/ echr-on-liability-of-isps-as.html (consultado el 8 de diciembre de 2014).

Information Society Services Act, of the European Parliament and of the Council of 8 June 2000 on Certain Legal Aspects of Information Society Services, in Particular Electronic Commerce, in the Internal Market ('Directive on Electronic Commerce'), http://eur-lex.europa.eu/LexU 
riServ/LexUriServ.do? uri=CELEX:32000L0031:en:HTML (consultado el 8 de diciembre de 2014).

JOHAN, Tere, "Estonian Supreme Court ruled in Favor of Leedo in the Dispute against Delfi", 10 de junio de 2009, http://www.baltic-course.com/ eng/Technology/?doc $=14708$ (consultado el 8 de diciembre de 2014).

MONGE, Yolanda, "Las mayores empresas de Internet piden a EEUU regular las leyes de espionaje", El Pais, 9 de diciembre de 2013, http:// internacional.elpais.com/internacional/2013/12/09/actualidad/1386 588911_791315.html (consultado el 8 de diciembre de 2014).

ONU, Asamblea General, Tercer Comité, "El derecho a la privacidad en la era digital", 26 de noviembre de 2013, https://www.un.org/News/Press/ docs/2013/gashc4094.doc.htm (consultado el 8 de diciembre de 2014).

REIDY, Pradaig, "European rulling Expels Trouble for Online Comment", 10 de octubre de 2013, http://www.indexoncensorship.org/2013/10/ european-ruling-spells-trouble-online-comment/ (consultado el 8 de diciembre de 2014).

SonTANI, Ashkan et al., "NSA uses Google Cookies to Pinpoint Targets for Hacking", The Washington Post, 10 de diciembre de 2013, http:// www.washingtonpost.com/blogs/the-switch/wp/2013/12/10/nsa-usesgoogle-cookies-to-pinpoint-targets-for-hacking/ (consultado el 8 de diciembre de 2014).

Tribunal de Justicia de la Unión Europea, Gran Sala, asunto Google France Sarl y Google Inc. contra Louis Vuitton Malletier SA (C-236/08), 23 de marzo de 2010, http://curia.europa.eu/juris/liste.jsf? num $=C-236 / 08$ (consultado el 8 de diciembre de 2014).

Others (C-324/09), 12 de julio de 2011, http://curia.europa.eu/juris/ liste.jsf?language $=$ en\&num $=C-324 / 09$ (consultado el 8 de diciembre de 2014).

, Tercera Sala, asunto Scarlet Extended SA vs. Société belge des auteurs, compositeurs et éditeurs SCRL (SABAM) (C-70/10) 24 de noviembre de 2011, http://curia.europa.eu/juris/liste.jsf?language $=$ en\&num $=C-70 / 10$ (consultado el 8 de diciembre de 2014).

"Víctimas colaterales", sección Editorial, El País, 11 de diciembre de 2013, http://elpais.com/elpais/2013/12/10/opinion/1386700261_735049.html (consultado el 8 de diciembre de 2014). 
Worstall, Tim, "Every Website that Accepts Comments Now has a European Problem", 10 de noviembre de 2013, http://www.forbes.com/si tes/timworstall/2013/10/11/every-website-that-accepts-comments-nowhas-a-european-problem/ (consultado el 8 de diciembre de 2014).

WyATT, Eduard y MiLler, Claire, "Tech Giants Issue Call for Limits on Government Surveillance of Users", The New York Times, 9 de diciembre de 2013, http://www.nytimes.com/2013/12/09/technology/ tech-giants-issue-call-for-limits-on-government-surveillance-of-users. html? smid=fb-share\&_r $=0$ (consultado el 8 de diciembre de 2014).

ZARA, Christofer, "Websites Liable for Anonymous Comments? European Court Upholds Defamation Case against Estonian News Portal", 11 de octubre de 2013, http://www.ibtimes.com/websites-liable-anonymouscomments-european-court-upholds-defamation-case-against-estoniannews (consultado el 8 de diciembre de 2014). 